\title{
News Not Noise: Socially Aware Information Filtering
}

\author{
Jonathan Melhuish \\ Advanced Interaction Group \\ School of Computer Science \\ University of Birmingham \\ Edgbaston, Birmingham \\ B15 2TT, UK \\ +44(0) 1214143744 \\ mail@orangejon.com
}

\author{
Russell Beale \\ Advanced Interaction Group \\ School of Computer Science \\ University of Birmingham \\ Edgbaston, Birmingham \\ B15 2TT, UK \\ +44 (0) 1214143729 \\ R.Beale@cs.bham.ac.uk
}

\begin{abstract}
An understanding of how people in social networks consume news media by and about their friends shows that information overload is soon going to be a major problem for many participants. Users dislike manually prioritizing their friendships to help organize this data, and this leads us to develop a new interface to help users to find the news that most interests them by providing a visual representation of social proximity, in which friends most visited and those most likely to be met offline we prioritized.
\end{abstract}

\section{Categories and Subject Descriptors}

J.4 [Computer Applications]: Social and Behavioral Sciences - psychology. H 5.2 [User Interfaces]: Prototyping. Screen Design, User-Centred Design

\section{Keywords}

Social networks, Facebook usage, information overload

\section{INTRODUCTION}

Online social networking services have dramatically risen in popularity in recent years, particularly amongst younger people [6]. These services facilitate multi-modal communication amongst users by making it easy to discover material created by friends and also to create, upload and publish content. This content may be textual, visual, or multimedia or may be another form of interaction between users such as playing a game.

Brief exploration of the user profiles on a social networking service reveals that these relationships are not only varied but vast in number. Many users seem to have several hundred "friends" in their online social network. Talking to users quickly reveals that it is quite unusual to ever delete a friend from one's online social network, so this number will presumably grow ad infinitum. Social psychology tells us that an individual's number of meaningful relationships stays relatively constant and small (around 20). This implies that a large proportion of the "friends" on the average social networking service's profile are more accurately acquaintances with which the user has no significant social bond, meaning that he may make little effort to maintain the relationship.

If this is indeed the case, then users will soon start to suffer from information overload, if they do not do so already. With many contacts, each producing news, the quantity of information flooding in to an individual will soon become unmanageable. In order to gain an understanding of this, we undertook a survey of Facebook users to understand more about

(c) The Author 2008.

Published by the British Computer Society their online relationships and how they consumed the media that their friends produce.

\section{FACEBOOK SURVEY}

A survey was sent to a set of 'friends' on Facebook, who are a varied collection of people - undergraduates, postgraduates, peers, family, and so on. There are related surveys by Joinson [5] and Ellison et al. [3], though our aim was not to undertake a massive survey of people, but to gain more insightful, deeper responses from a more limited set. The results are discussed below. Where a correlation between two variables is given, this indicates that a Pearson Correlation was found with a significance level of at least 0.05 . In the case of comparisons between the ratings given to different categories, the Wilcoxon Signed Rank Test was used. As multiple hypotheses (pair-wise comparisons) were being tested, the probability of finding a hypothesis to be true by chance is increased. A higher significance threshold of 0.01 was used to compensate for this. The data were analysed using SPSS. 148 of one of the author's Facebook friends were asked to participate in the study, of whom precisely half responded. There were slightly more male respondents $(58 \%)$. The average age was 24 , with a sharp peak around 22 (the first author's age). The standard deviation was 5.23 (to 3 s.f.).

Usage

Almost half $(45 \%)$ of respondents reported logging into Facebook several times a day. Only 5\% of respondents logged into Facebook less often than "every few days". Note, however, that the invitation to participate in the survey was sent via Facebook, so those who log in less frequently may feel less compelled to respond.

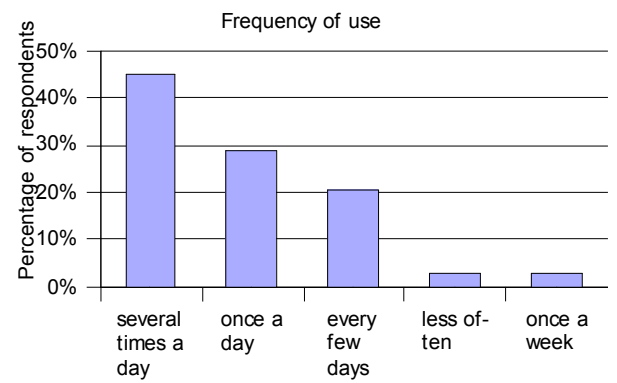

Figure 1: Frequency of Facebook logins

One respondent commented that the frequency of his usage varies dramatically:

"Ask me a month ago and I was on several time a day and updating my status all the time. Now I update status once a week! and don't even log on every day."

A large majority of respondents (86\%) logged in for half an hour or less per session. The average reported session duration was 23 minutes, with a median of 15 minutes and a standard deviation of 22.7 . 
Average session duration (minutes) by frequency

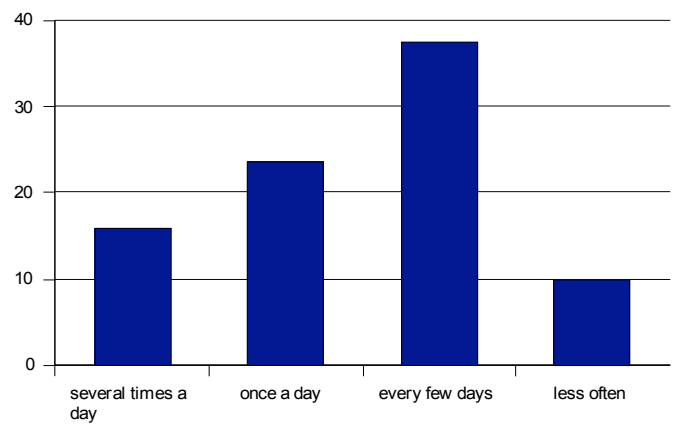

Figure 2: Average session times, grouped by login frequency

Average reported session duration increased as the login frequency decreased, as might be expected, apart from for those users who logged in less often than "every few days".

The survey asked respondents to rate the importance of several key functions of Facebook on scale from 1 to 5 stars, where 1 is labelled "not important" and 5 "very important", but the meaning of intermediary ratings is not prescribed. The most important reason cited for using Facebook was to keep up to date with their current friends' lives, with an average rating of 3.9 and the vast majority (89\%) giving this reason three stars or higher. It may seem surprising that they would want to communicate via online social networks with people they frequently meet face-to-face and communicate with through other media, but it appears that people use social networking services more as a way to view information about their friends' lives that their friends may not communicate to them directly. Indeed, in the case of photographs, where Facebook allows users to easily see photographs in which their friends appear, somebody they do not know may well have created the media. It is very unlikely that this type of sharing would happen if it were not facilitated by the social networking platform. In close second and third places respectively were contacting friends (3.7 stars average) and rediscovering old friends (3.6 stars), with around three-quarters $(77 \%)$ of respondents giving three stars or higher for each. There was no statistically significant difference between these three reasons.

These results suggest that people are using online social networks to replace their address book. This overcomes difficulties with out-of-date contact details that other systems typically suffer from. Contact via social networking platforms also has different overtones to that of other communication media, especially writing on a user's Facebook "wall" essentially, writing a message that is intended for that user but can be read by anyone who knows that user. This might be roughly equated to a comment made face-to-face to that person in a public place, where other peers will moderate comments. This is often how friendships in the real world are formed before any one-to-one meetings are considered appropriate.

However, respondents were widely agreed that Facebook was not a place for making new friends, with two-thirds $(67 \%)$ giving just one star and almost all (93\%) giving three stars or less. Men were significantly more likely to give a higher score than women, with an average rating of 1.79 , compared to 1.59 for women. It appears that meeting new friends is still an activity that mostly happens offline. However, sites such as HospitalityClub.org, Meetup.com and Match.com suggest that many do use the Internet to find new people to meet, when there is some shared interest or motivation. It seems that Facebook is not frequently used in this way, although there are some indications that third-party applications (such as "Are You Interested?", a simple dating application with around 500,000 daily active users) are starting to fill this void.

\section{Number of friends}

There was a wide variance in the number of friends that respondents reported having on Facebook, from just 2 to 883 . $91 \%$ of respondents had over 50 friends; the number of responses trails off quickly after 350 , with only $10 \%$ of respondents reporting having over 350 friends. The average was 212 and the median was 168 with a standard deviation of 159 (to 3 s.f). Several respondents commented on how "friends" is perhaps an inaccurate description of the contacts listed on their Facebook profile, for example:

"A lot of the people are on my list because they like to add absolutely everybody they have ever known even if they have no intent of talking to [me], but [they] want to look at what [I] do I guess."

and, more succinctly:

"God. So many 'friends' are actually zombie subacquaintances!"

One respondent mentioned that they thought there was some peer pressure to add as many friends as possible:

"Facebook sometimes comes across as a pissing contest to see who has more friends. Lots of people have something like 300 friends, despite studies showing that 100 people is the maximum number of real social interactions you can havet... As a rule, I generally only add people who I see regularly, with some exceptions."

("Probably a reference to "Dunbar's number", 150, a suggested average size of human social group that is manageable [2].)

The average number of friends added by users in the last 2 weeks was 3.7 (s.d. 5.01), suggesting that the average user's number of friends is growing by $45 \%$ per annum, as previous interviews have suggested that they rarely remove friends. There seems to be a significant reluctance to delete friends as it seems slightly confrontational. One user suggested that he didn't like to voluntarily block himself from accessing personal information about somebody:

"I know I should delete some of my "friends" but you never know when you might need to creep around their page to see what they have been up to!"

The maximum (34) came, unsurprisingly, from the user with the greatest number of friends (883). $20 \%$ of respondents stated that they had not added any friends in this period.

The survey asked respondents to classify 50 of their Facebook friends into five categories (close friend you see regularly; old friend who not seen regularly; new friend, seen regularly; don't know well; family). However, many respondents apparently misunderstood the instruction and classified more than 50 friends. In these cases, it was assumed that they had classified all of their friends. Responses with no friends categorized were assumed to have not completed the question. The category into which users placed most of their friends on average was old friends who they do not see regularly, with an average of $18 \%$ of their friends. In second place were current friends, with $10 \%$. The other categories had less people in, with an average of $13 \%$ in total. Women had significantly more close friends than men, twice as many on average $(12 \%$ vs. $6 \%)$. Respondents only classified an average of $41 \%$ of their friends into the suggested categories, suggesting that the categories were not very comprehensive, despite feedback to the contrary when the survey was initially being tested. One respondent, who categorized $62 \%$ of his friends, confirmed in a comment that the remainder of his friends don't fit into the given categories. Some respondents suggested additional categories in their comments:

"The missing category... is a friend who you contact regularly but don't see regularly. That would be about 25 of the 50 people." 
It is also possible that as this question was quite timeconsuming and was placed at the end of the questionnaire that some respondents lost patience with classifying their friends. It may also indicate that respondents found it difficult to classify their friends. There were several comments from those who initially tested the questionnaire that indicated that they found this question demanding, and also that they felt awkward and uncomfortable in having to categorize friends in this way.

\section{News Feed}

The "News Feed" is Facebook's name for the aggregated view of news by and about all of the user's friends. This view is the first page that users see when logging in, and can be easily reached from any other page by clicking on the Facebook logo. This prominent position within the information architecture implies that Facebook see this feature as a key means of navigation through the service's content. However, interest in the aggregated view of all news about the user's friends (the "News Feed") was very low; the majority of respondents reported finding one-quarter or less of the News Feed items interesting, with just $14 \%$ of respondents reporting interest in over half of their News Feed items. It would seem a plausible hypothesis that users would add their closest friends to their online social network first and that a small number of friends should indicate that the user is more selective about who they add. If this were true, a user with fewer friends would be interested in a greater proportion of the items in their News Feed. However, we found no significant correlation between these factors.

Photographs were widely considered the most interesting type of social media, with an average rating of 3.9 stars and the vast majority $(92 \%)$ giving three stars or more. Friends' intention to attend events was generally considered interesting, with around half $(54 \%)$ giving three or four stars. Friend's actions on Facebook were widely considered the least interesting, with almost all (96\%) giving three stars or under. Interest in the News Feed was significantly related to interest in profile changes and friends actions (e.g. joining a group, using an application). This suggests that other stories may currently be over-represented in the News Feed, contributing to the low average level of interest.

Respondents reported equal interest in close friends they see regularly and old friends who they don't see regularly, with an average star rating of 3.4 for each. There is no significant difference between any of the four most interesting types of friends. Respondents were significantly more interested in those they don't know well but who they see regularly than those they don't see regularly, with the most respondents choosing 3 stars and 2 stars respectively. This suggests the hypothesis that a user's interest in other Facebook users is influenced by the likelihood of the users meeting off-line. Younger users were significantly more interested in new friends. Almost all users (91\%) gave star ratings of 3 or below for those friends who don't fit into the suggested categories, which is significantly lower than all other categories. This confirms that the categories chosen have a strong relation to their level of interest in a given user.

The same 148 people were invited to participate in a second survey, to which 20 responded (13.5\% response rate). Participants were asked whether they have used the feedback buttons on the News Feed (which have been removed since the research was conducted). Three-quarters of respondents stated that they had never used them, $10 \%$ had used them once or twice and $15 \%$ had used them "sometimes". No respondents stated that they used them regularly. Of those respondents who had not used the feedback buttons, the majority (60\%) of respondents said that they hadn't noticed them. This is understandable as the icons were very small and faint. About a quarter $(27 \%)$ of respondents didn't feel the need to use them and $13 \%$ didn't understand their function. Of those that had used the feedback buttons, none felt that they'd had any effect on which items were shown in the News Feed.

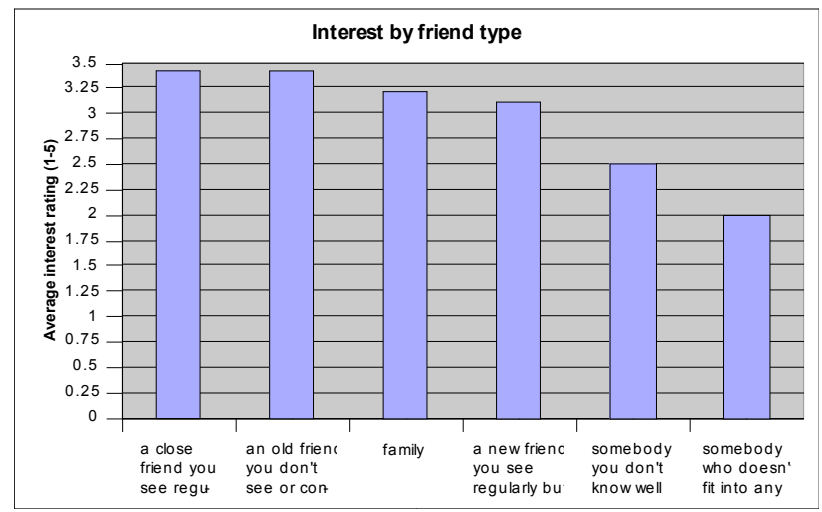

Figure 3: Likely interest in news item, by category of friend

\subsection{Key findings}

Usage of Facebook is generally fast and frequent, similar to email. This does not normally allow enough time to consume all news by all the user's friends, so tools to help the user find the most interesting news are important. Yet the satisfaction with the aggregated news feed provided by Facebook, which is typical of those provided by social networking services, is very low. This confirms that there is an important problem in this area. Keeping up-to-date with current friends and reconnecting with old friends are the two most important reasons for using Facebook, but although they seem like similar tasks, they differ dramatically in the "amount of detail" the user is interested in - the user is likely to want to have their attention drawn to a smaller number of stories about old friend's lives, that are of more significance. Users have almost twice as many "old" friends as "current" friends: displaying a random selection of news will show more from old friends. The proportion of old friends will increase as the user adds new friends, as deleting friends seems to happen rarely (as previously discussed) and research shows that the number of close friends at any one time stays relatively constant, possibly limited by social cognitive abilities [7]. Interviewees and survey respondents both admitted that many of those on their "friends" list were not people that they knew well. This may be attributed to the apparent social stigma associated with refusing friend requests or deleting friends from the list. Respondents reported being significantly less interested in these people.

\section{REDESIGNING THE NEWS FEED}

The survey attempted to get potential users to classify friends by the type of relationship they share, with only limited success. Another way to look at different friendships is to look at the context in which the friendship develops, such as the workplace, place of study or a club or organisation.

We used a force-directed visualization, where individuals who share friends or co-appear in photographs are shown closer together, and this reveals definite groups corresponding to different social contexts, such as classmates or work colleagues. This suggests that those who share friendships are also share other similarities, which is supported by Galton [4], who obtained correlational data showing that in married couples, spouses are similar in many respects. In turn, the sharing of stories and information is important in bringing people together. Brown \& Duguid [1] argue that rather than the information 
itself, it is the shared interpretation of these stories that makes individuals feel closer to one another, and that this process of developing a common framework is critical for collaboration around shared information. Although they discuss this in the context of a team of work colleagues, it is easy to see parallels with many social situations, particularly where some group activity is being performed with some shared goal. Similarity, therefore, has implications both for the estimation of social proximity, and hence level of interest in news about that person, and how the information should be presented. Stories from those within a social group will make more sense when told in the context of stories from other members of that group.

Perhaps by representing these ideas of social context and social proximity within the user interface, we can help users to filter social information better. Several designs were iterated through and one developed into a digital prototype. This design represents the user's friends as profile photographs in concentric circles, with their closest friends (i.e. most likely to meet offline) shown towards the centre and similar friends shown adjacent to each other, such that the angle represents social context. Users can hover over the profile photograph in order to reveal what story types are available, and click in order to reveal the stories.

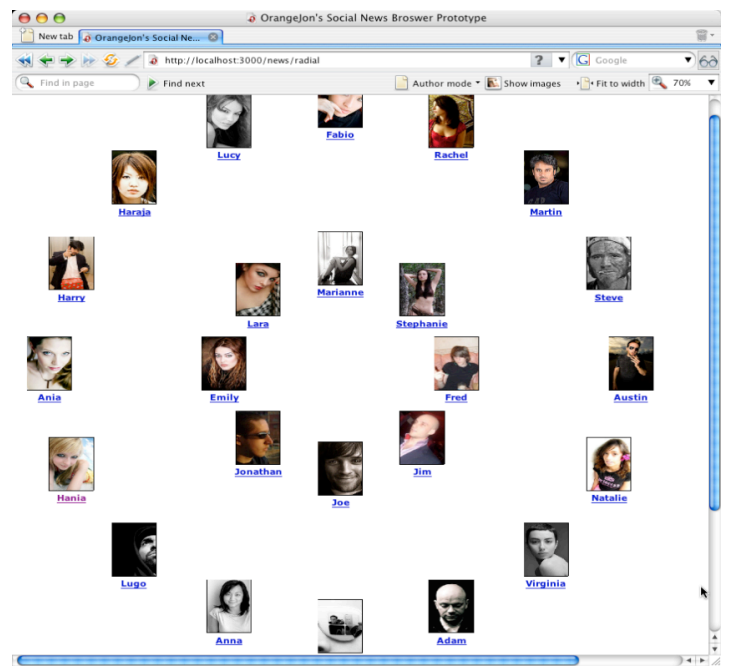

Figure 4: Screenshot of prototype friend and news viewer

A screencast video was prepared showing the key elements of the prototype interface in action and explaining the significance of the layout (online at http://youtube.com/watch? $v=4 \mathrm{AbWpW}$ $w u u k$ ). The same audience as before were surveyed again regarding this new potential interface. Out of the 148 people invited to participate in the survey, 11 responded $(7.4 \%$ response rate). Three respondents expressed reservations about the amount of interaction required:

"If I had to deliberately choose specific people to click on to see any news, I probably wouldn't bother"

"I find it quite boring to have to click on photos of everybody you're interested in to see their news."

"I think it could be quicker and easier to scan through a list of news feeds on a page."

The concept of organising friends by estimated social proximity was widely welcomed:

"Many of the people on my list are friends from school who I don't need to know about day to day"

"...it would make navigating through news stories a bit easier." "the fact that the computer could choose to show you news feed from your most clicked profiles is definitely a good idea"

One user disliked the idea of removing some of the serendipity of the News Feed: "narrowing down the news feed could take away some of the surprising to find out something for someone that you don't expect."

There was significant disagreement about how the design would function with different numbers of friends displayed, with some respondents believing that it would be of most benefit to those with large numbers of friends but others feeling that the display would become ineffective when large numbers of friends needed to be displayed. It is possible that this confusion was caused by the use of too few friends in the prototype, which meant that all of them could be displayed quite comfortably on the display: conceptually, large numbers can be accommodated in ever-expanding circles, closest friends nearer the centre.

\section{CONCLUSIONS}

The fundamental advantages of social networking services stem from their geographically agnostic, asynchronous connection of people, and the electronic representation of this social structure. For example, the problem of displaying socially-relevant digital media is effectively achieved by displaying to the user content that is either published by or concerns their friends. This and related research has shown that people are using social networking services to support a wide variety of relationships. However, the ability of the social networking service to display interesting news is being undermined by the over-simplistic underlying social model.

The answer does not seem to be to ask users to manually classify their friends. Although respondents considered that the proposed categories described the most interesting individuals in their online social network, on average they only managed to classify a minority of their friends, giving little clue as to their relationship with the remaining others. Additionally, this task was found to be demanding and time consuming.

Instead, we propose that user interfaces may be devised that draw on machine learning techniques to assist the user in filtering and prioritizing social media. These techniques allow a far more complex and descriptive social model to be constructed, potentially with little extra effort on the user's part. Several interface designs were iterated through, one of which was developed into a digital prototype which elicited positive initial reactions from social network users. This prioritized people based on the likelihood you would meet them offline as well as those whose profiles were clicked the most.

\section{REFERENCES}

[1] Brown, J. and Duguid, P. The Social Life of Information. Harvard Business School Press, 2000.

[2] Dunbar, R. The Tipping Point. Wheeler Publishing, 2003. [3] Ellison, N., Steinfield, C. and Lampe, C., Spatially Bounded Online Social Networks and Social Capital: The Role of Facebook. in Annual Conference of the International Communication Association, (2006).

[4] Galton, F. Hereditary genius: An inquiry into its laws and consequences. http://galton.org/books/hereditary-genius/, accessed on 26th March, 2008

[5] Joinson, A.N., Looking at, looking up or keeping up with people?: motives and use of facebook in Proceeding of the twenty-sixth annual SIGCHI conference on Human factors in computing systems (Florence, Italy 2008), ACM, 1027-1036.

[6] Ofcom. Social Networking : A quantitative and qualitative research report into attitudes, behaviours and use. http://www.ofcom.org.uk/advice/media_literacy/medlitpub/med litpubrss/socialnetworking/report.pdf

[7] Stiller, J. and Dunbar, R.I.M. Perspective-taking and memory capacity predict social network size. Social Networks, 29 (1). 93-104. 